\title{
Melting line of aluminum from simulations of coexisting phases
}

\author{
J. R. Morris, C. Z. Wang, K. M. Ho, and C. T. Chan \\ Ames Laboratory-U.S. Department of Energy, Department of Physics and Astronomy, \\ Iowa State University, Ames, Iowa 50011
}

(Received 9 July 1993; revised manuscript received 16 September 1993)

\begin{abstract}
We have performed simulations of coexisting liquid and solid phases of aluminum as an efficient way of mapping out the coexistence line. This technique is convenient, as it does not require complicated free energy calculations for the different phases, but simply allows the system to equilibrate to a coexistence point. By altering the simulation volume and/or energy, a new coexistence point is found. The calculated melting temperature is lower than previous results for the identical model; we suspect that this difference is due to the difficulty of calculating the free energy of the liquid phase, leading to inaccuracies in the previous work. A thorough examination of several different system sizes, from 1024 to 65536 particles, indicates that the results are only weakly dependent upon the system size.
\end{abstract}

\section{INTRODUCTION}

Although molecular dynamic and Monte Carlo techniques have been used for many years, it is still difficult to make accurate theoretical predictions concerning the melting temperature of real materials. Part of the difficulty is accurately modeling the interactions in real materials; however, with the recent advances in empirical many-body potentials of metals, ${ }^{1,2}$ tight-binding molecular dynamics, ${ }^{3}$ and first-principles calculational techniques, ${ }^{4-6}$ simulations of materials are becoming increasingly realistic. Nevertheless, determining the melting temperature for a given model may still be quite difficult due to hysteresis near the first-order transition; simulated crystals with periodic boundary conditions can be overheated to temperatures much higher than the equilibrium melting point - for the system we consider below, we could heat the solid phase to temperatures over $500 \mathrm{~K}$ above the melting temperature without the system melting. Thus, even for simple models, the location of the melting line may be difficult to calculate accurately.

One approach to avoiding hysteresis is by calculating the free energies of the solid and liquid phases. At the transition, the free energies are equal; thus, an accurate calculation of the free energies can give the transition temperature. The difficulty is in calculating the free energies of the different phases: usually, this is done using the relation

$$
F=F_{0}+\int_{0}^{1} d \lambda\left\langle V-V_{\lambda}\right\rangle_{\lambda},
$$

where $F$ is the true free energy calculated from the potential energy $V$, and $F_{0}$ is the free energy of some exactly solvable system corresponding to a potential $V_{0}$. This relation may be derived simply from the canonical ensemble; note that the average in Eq. (1) is calculated with respect to the potential $V_{\lambda}=\lambda\left(V-V_{0}\right)$. The average in the integrand may be calculated for a given value of $\lambda$ by performing a simulation of the system with the dynamics governed by the potential $V_{\lambda}$. Using a series of these values, an approximate value of the free energy may be calculated. Once the free energy of the system is known at a given temperature, a similar process may be followed to find the free energy at other temperatures using the relation

$$
\frac{F}{T}-\frac{F_{0}}{T_{0}}=\int_{T_{0}}^{T} d \tau \frac{E(\tau)}{\tau^{2}} .
$$

While the use of Eq. (1) is straightforward in principle, there are a number of difficulties and drawbacks in this technique. For the solid phase, the reference system is usually chosen to be some harmonic or Einstein solid. ${ }^{7,8}$ For the liquid phase, however, the situation is more difficult, due to the lack of a convenient reference system. The usual choice of a reference point is an ideal gas, which may be reached by isothermally expanding the system volume. However, the integration path in Eq. (1) must be reversible, i.e., no phase transition may take place. Performing such an expansion is then unsuitable, as it will generally take the system through a liquid-vapor transition. One approach $^{8,9}$ is to turn off the attractive part of the interactions and then perform a slow expansion to a weakly interacting gas. This avoids the liquid-vapor transition and is therefore (in principle) a reversible path.

Another approach is to determine the free energy of the liquid phase directly by performing a grand-canonical Monte Carlo simulation of the liquid at a given temperature, which may be used to calculate the chemical potential and therefore the free energy at that temperature. ${ }^{10,11}$ Then Eq. (2) may be used to calculate the free energy at new temperatures. This technique also may be computationally expensive, as the reference free energy must be known quite accurately.

Whatever the technique, calculating the free energy using Eqs. (1) and (2) requires a series of calculations, which takes up much computer time. Further, it has been 
pointed out ${ }^{11}$ that an error in the Gibbs free energy of $0.01 \mathrm{eV} /$ atom may produce a corresponding error in the melting temperature of $100 \mathrm{~K}$. Thus, much care must be taken to ensure that the free energies are accurate when using this approach.

Experimentally, hysteresis is not a problem - in fact, even slightly superheating solids is usually quite difficult. This is due to heterogeneous nucleation: defects act as nucleation sites for the liquid phase as the crystal is heated. These defects may be vacancies, dislocations, or surfaces; in fact, some surfaces become disordered well below the bulk melting temperature. Surface premelting has been examined using simulations for a number of materials, including aluminum ${ }^{12}$ and gold. ${ }^{13,14}$ Thus, introducing a surface,${ }^{12,13}$ a vacancy, ${ }^{15}$ or some other crystalline defect into the simulation may help nucleate the liquid phase. However, it may still be difficult to obtain reliable estimates of the transition temperature. One must differentiate between local disorder or premelting near the defect, and bulk melting.

A more direct approach ${ }^{13}$ to finding the transition temperature is to avoid the nucleation problem altogether, i.e., by simulating coexisting phases and allowing the system to evolve to equilibrium. If the equilibrium system contains both solid and liquid phases, then the system will be at a melting point. This approach is suitable for both experimental and theoretical studies. Molecular dynamic (MD) techniques are particularly useful for this approach, due to the fact that total energy is conserved in conventional MD schemes. To understand how this helps the system evolve toward equilibrium, consider a system with a phase boundary. If the system as a whole is at a temperature slightly below the melting point, then some portion of the liquid phase will solidify, generating the appropriate latent heat. Because the system is closed, this heats up the system towards the melting point. Similarly, if the system is above the melting temperature, the latent heat required to melt the solid will cool the system. The pressure of the system will also tend to equilibrate; thus, the system will evolve toward an equilibrium phase. There is no difficulty in nucleating either the liquid or solid phases, as the interface assists in the nucleation for the melting or solidification process.

Once one point on the phase diagram is known, the system can be perturbed slightly, by altering the density and/or the total energy, and a new equilibrium point will be established. The presence of a boundary between the different phases helps avoid difficulties with hysteresis the system can equilibrate quickly to the new conditions. This allows for a rapid, systematic exploration of the phase diagram, which is a very strong advantage of this technique.

In this paper, we examine this technique, using an embedded-atom model (EAM) of aluminum. ${ }^{1,2,8} \mathrm{We}$ have chosen this model because the melting temperature of this model at zero pressure has been previously studied ${ }^{8}$ by calculating the free energies of the solid and liquid phases using Eq. (1) and because of the simplicity of the EAM model compared to more realistic simulation techniques. We have calculated the melting temperatures for $P<40 \mathrm{kbar}$, and find that the melting temperature at
$P=0$ is significantly lower than the value obtained in the work by Mei and Davenport. ${ }^{8}$ We believe that our technique is more reliable in practice, as it is easier to estimate the errors in the calculations. From the calculated coexistence line, we have found that the change in entropy at the transition is in good agreement with the experimental value.

\section{MODEL AND TECHNIQUE}

As mentioned in the Introduction, we use the EAM model of aluminum proposed by Mei and Davenport. ${ }^{8}$ This potential takes the form

$$
E_{\text {total }}=\sum_{i} F\left(\rho_{i}\right)+\frac{1}{2} \sum_{i, j} \phi\left(r_{i j}\right), \quad \rho_{i}=\sum_{j \neq i} f\left(r_{i j}\right)
$$

where $F(\rho)$ is an analytic many-body force term dependent upon the local electron density $\rho_{i}$, which is calculated from the polynomial $f(r)$. A cutoff function is used so that the potential goes smoothly to zero at $r_{c}=5.58441 \AA$. We shall not discuss the motivation of the potential here, except to note that it is an analytic many-body potential fitted to the structural properties of fcc $\mathrm{Al}$, including the cohesive energy, lattice constant, elastic constants, and unrelaxed vacancy formation energy. We refer to the original paper by Mei and Davenport for further discussion of the potential.

Most of the simulations were performed for 1024 particles; however, we have also performed simulations with 4096,8192 , and 65536 particles, to test that the results are not sensitive to system size. These finite-size tests will be discussed later. Initially, each simulation had half of the particles in the solid phase and half in the liquid phase. The two phases had previously been equilibrated separately at a temperature of approximately $800 \mathrm{~K}$, the melting temperature predicted by Mei and Davenport. The total energy was adjusted by calculating the initial potential and kinetic energies, then scaling the velocities to adjust the system to the desired total energy. The simulations consisted of numerically integrating Newton's equations of motions, with periodic boundary conditions. As a result, the total energy and the volume were fixed during each simulation. The simulations ran for a total of at least 20000 time steps of $2.654 \times 10^{-15} \mathrm{sec}$.

The temperature was calculated using

$$
\frac{3}{2} N k_{B} T=\left\langle\sum_{i=1}^{N} \frac{1}{2} m v_{i}^{2}\right\rangle
$$

in the simulations, a time average of the kinetic energy over either the last 5000 time steps was used. The results for the average temperature and pressure were nearly converged after the initial 5000 time steps: the results for the following 5000 time steps were very similar, indicating that this is a sufficient initialization time. Similarly, the pressure was calculated using the instantaneous forces $\mathbf{F}_{i}$ and positions $\mathbf{r}_{i}$ in the virial equation 


$$
P V=N k_{B} T+\frac{1}{3}\left\langle\sum_{i=1}^{N} \mathbf{F}_{i} \cdot \mathbf{r}_{i}\right\rangle
$$

For EAM potentials, this may be written as ${ }^{11}$

$$
P V=N k_{B} T-\frac{1}{3}\left\langle\sum_{i, j \neq i}\left[F_{i}^{\prime}\left(\rho_{i}\right) \rho_{j}^{\prime}\left(r_{i j}\right)+\frac{1}{2} \phi^{\prime}\left(r_{i j}\right)\right] r_{i j}\right\rangle .
$$

For a constant volume simulation with different phases present, the pressure may be anisotropic, i.e., the system may be under some effective tension or compression. We chose the simulation box size and shape so that the average pressure was nearly isotropic.

\section{RESULTS}

Several possible final configurations are shown in Fig. 1 for the 1024 particle system. Figure 2 shows the final configuration of 12000 particles from the $N=65536$ system, representing a cross section of $\sim 13 \AA$. The particle positions have been projected onto the $(010)$ or the (001) plane of the original crystal; the initial interfaces were (100) faces of the fcc crystal. The periodic boundary conditions and the chosen geometry cause there to be two interfaces between the different phases. The geometry of the system was chosen such that the system was approximately four times longer along the $x$ direction than along the $y$ or $z$ directions for $N=1024,8192$, and 65536 , and approximately twice as long for $N=4096$; see Tables I and II for the actual dimensions used. This geometry increased the number of crystalline layers that were initially between the interfaces, relative to a cubic geometry. This helped prevent the system from completely transforming to a single phase. In Fig. 1, the open circles represent particles, which were initially in the solid phase, and the solid circles indicate particles initially in the liquid phase. As seen in Fig. 1(b) and Fig. 2, there may be regions of both solid and liquid phases remaining after the simulation was completed. This stable coexistence demonstrates that the system is at an equilibrium melting temperature and pressure.

After each simulation, it was verified that there were still two phases coexisting in the system, i.e., the final stable equilibrium point roughly corresponded to the situation shown in Figs. 1(b) and 2 with both solid and liquid regions present. We have calculated the instantaneous pair correlation function $g(r)$ for a solid portion of the system following the simulations. This function is shown in Fig. 3, along with the distances of the neighboring atoms for a fcc lattice with the appropriate lattice constant. The function was calculated for $r \leq 8$ $\AA$, enabling us to examine the peaks corresponding to (a)

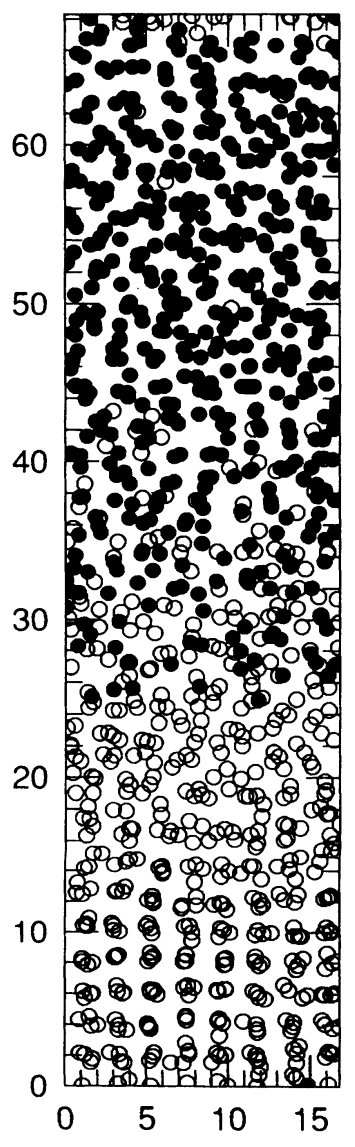

(b)

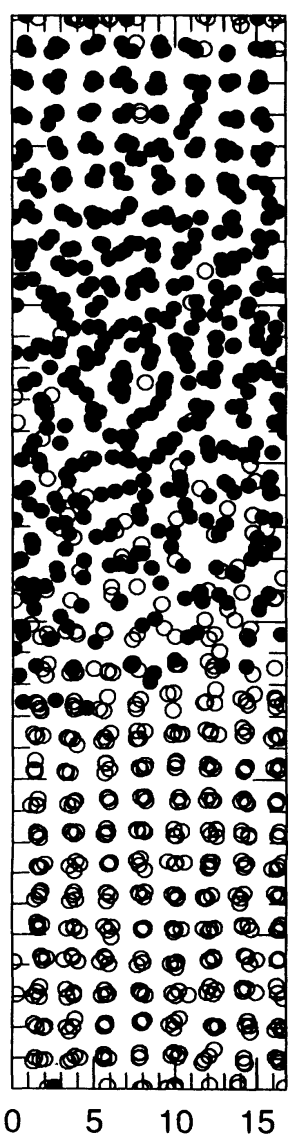

(c)

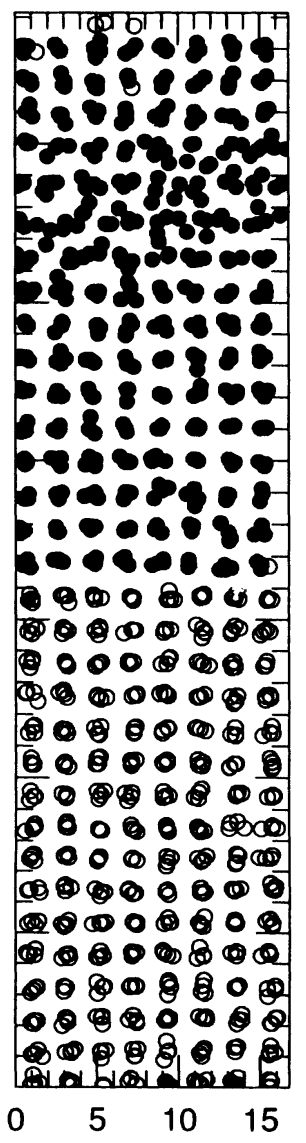

FIG. 1. Possible final configurations after equilibrating the system. The particle positions have been projected onto the $x-z$ plane. Open circles indicate atoms that were initially in the solid phase; closed circles indicate atoms initially in the liquid phase. Distances in angstroms are indicated on the axes. (a) Example of a system that has almost completely melted. (b) System in which some of the solid phase has melted and some of the liquid phase has solidified. (c) System that has almost completely crystallized. 
seven shells of neighboring atoms. The peaks are fairly broad, consistent with the system being near the melting temperature. All peaks appear in their appropriate positions, with the intensity consistent with the number of neighboring atoms at each distance, except for the sixthneighbor shell. This peak should have a low intensity, due to the fact that there are only eight neighbors in this shell. We believe that the peak cannot be seen due to the low intensity and due to the width and intensity of the peak for the seventh-neighbor shell. Thus, we conclude that the structure is completely consistent with that of a fcc solid, demonstrating that the solid portion has retained its bulk properties.

In several occasions the 1024-particle system completely transformed within the first 10000 time steps; however, all systems that remained in a two-phase state for the first 10000 time steps also maintained the coexistence for the next 10000 steps. This indicates that the initial 10000 steps of equilibration were sufficient. The results obtained for the 1024 system were used to predict

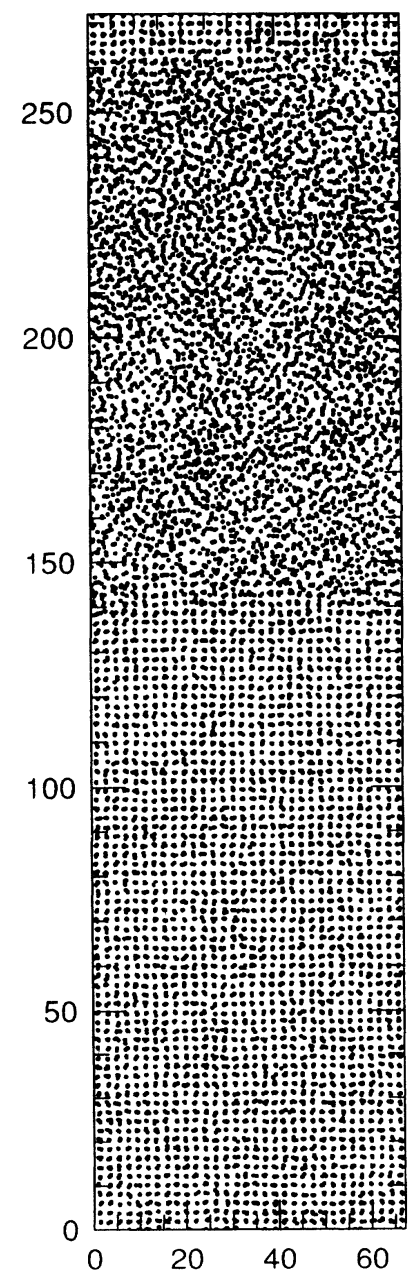

FIG. 2. Final configuration of 12000 atoms from the $N=65536$ system after equilibrating the system. $(\sim 13 \AA$ cross section of the total system is shown.) Again, the particle positions have been projected onto the $x-z$ plane. Distances in angstroms are indicated on the axes. geometries and energies for the larger systems; thus, we never observed a larger system completely transforming.

In Tables I and II, the geometries, energies, and average temperatures and pressures are listed for our simulations. Simulations in which the system completely transformed to a single phase are not included. Figure 4 shows the resultant pressure-temperature phase diagram, along with a fit of the $N=1024$ data to the form $T=T_{0}+\alpha P+\beta P^{2}$. The fit is quite good and is in complete agreement with the data from the (unfitted) results for the larger systems. The fitted transition temperature at $P=0$ is $T_{0}=724 \mathrm{~K}$, with an estimated error of $10 \mathrm{~K}$. We also find $\alpha=23.3 \mathrm{~K} / \mathrm{kbar}$ and $\beta=-0.15$ $\mathrm{K} /\left(\mathrm{kbar}^{2}\right)$. Although there is some scatter in our data, the results clearly indicate a melting temperature that is considerably lower than the value $T_{m}=800 \pm 10 \mathrm{~K}$ predicted by Mei and Davenport ${ }^{8}$ for the same model. We suspect that this discrepancy is due to some inaccuracy in the free energy of the liquid phase in the paper by Mei and Davenport, ${ }^{8}$ as we have separately calculated the free energy of the solid phase, and obtain results ${ }^{16}$ close to those of Mei and Davenport. Note that both calculations produce a significantly lower temperature than the experimental value of $T_{m}=933 \mathrm{~K}$; an inaccurate melt-

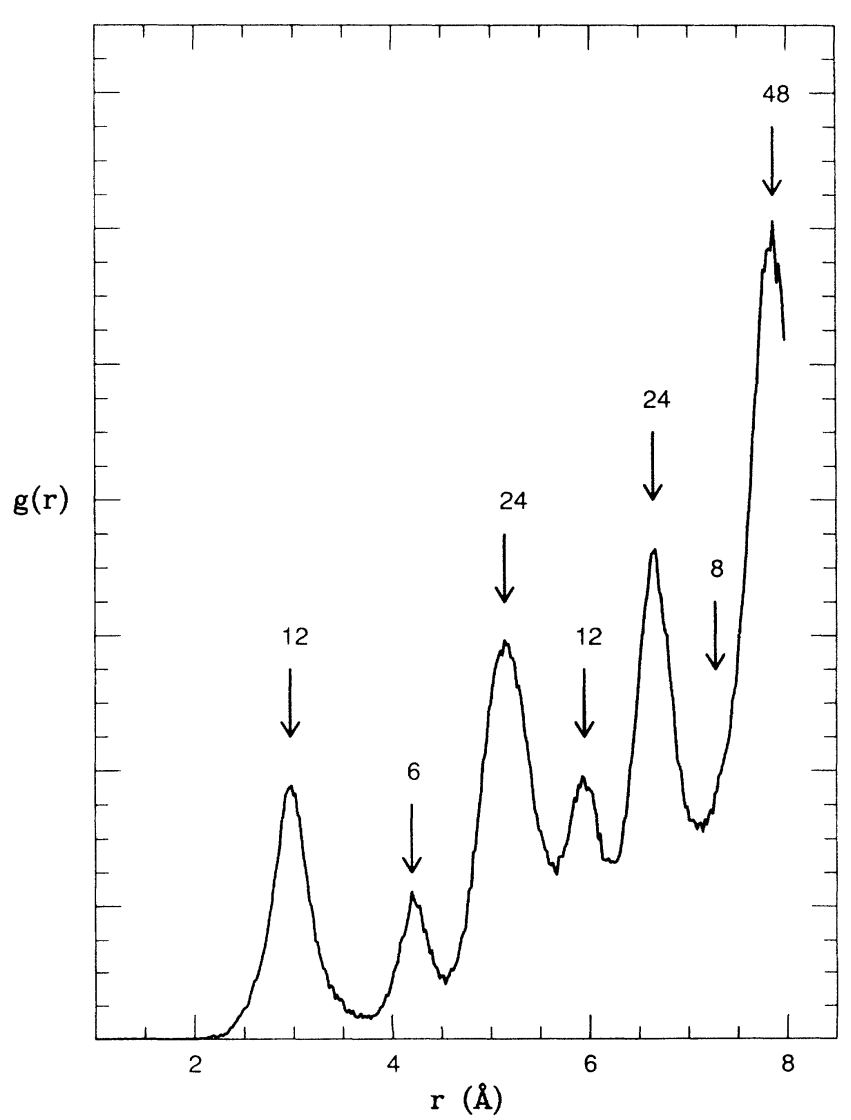

FIG. 3. Instantaneous pair correlation function $g(r)$ for the solid portion of the $N=65536$ sample after the simulation. The peak positions are consistent with the positions of the neighboring shells of a fcc solid with a lattice constant $a=4.2$ $\AA$, as indicated by the arrows. The appropriate number of atoms in each shell is also shown, to show that the intensities are also consistent with a fcc lattice. 
TABLE I. Coexistence melting temperature and pressures, found from individual simulations of 1024 particles. The total energy/atom and the box size for each simulation is also given. Note that the cohesive energy of the fcc crystal at $T=0$ is $E_{c}=3.4 \mathrm{eV}$.

\begin{tabular}{rccc}
\hline $\begin{array}{c}\text { Pressure } \\
(\mathrm{kbar})\end{array}$ & $\begin{array}{c}\text { Melting temperature } \\
(\mathrm{K})\end{array}$ & $\begin{array}{c}\text { Total energy/atom } \\
(\mathrm{eV})\end{array}$ & $\begin{array}{c}\text { Box size } \\
\left(\AA^{3}\right)\end{array}$ \\
\hline 0.1 & 721 & -3.125 & $68.30 \times 16.80 \times 16.80$ \\
1.1 & 750 & -3.120 & $68.00 \times 16.80 \times 16.80$ \\
2.2 & 786 & -3.115 & $68.10 \times 16.75 \times 16.75$ \\
4.7 & 827 & -3.110 & $67.60 \times 16.70 \times 16.70$ \\
6.2 & 858 & -3.099 & $67.60 \times 16.67 \times 16.67$ \\
8.5 & 891 & -3.095 & $67.30 \times 16.60 \times 16.60$ \\
9.1 & 943 & -3.094 & $67.20 \times 16.57 \times 16.57$ \\
9.8 & 945 & -3.093 & $67.15 \times 16.55 \times 16.55$ \\
11.3 & 969 & -3.090 & $67.00 \times 16.50 \times 16.50$ \\
12.2 & 978 & -3.080 & $67.00 \times 16.50 \times 16.50$ \\
14.0 & 1017 & -3.073 & $66.90 \times 16.45 \times 16.45$ \\
16.0 & 1069 & -3.065 & $66.70 \times 16.40 \times 16.40$ \\
19.2 & 1122 & -3.055 & $66.55 \times 16.30 \times 16.30$ \\
22.1 & 1159 & -3.045 & $66.50 \times 16.20 \times 16.20$ \\
25.5 & 1219 & -3.035 & $66.30 \times 16.10 \times 16.10$ \\
28.7 & 1281 & -3.025 & $66.15 \times 16.00 \times 16.00$ \\
32.2 & 1305 & -3.015 & $66.00 \times 15.90 \times 15.90$ \\
32.5 & 1338 & -3.010 & $66.00 \times 15.90 \times 15.90$ \\
37.7 & 1392 & -2.980 & $65.80 \times 15.80 \times 15.80$ \\
\hline \hline
\end{tabular}

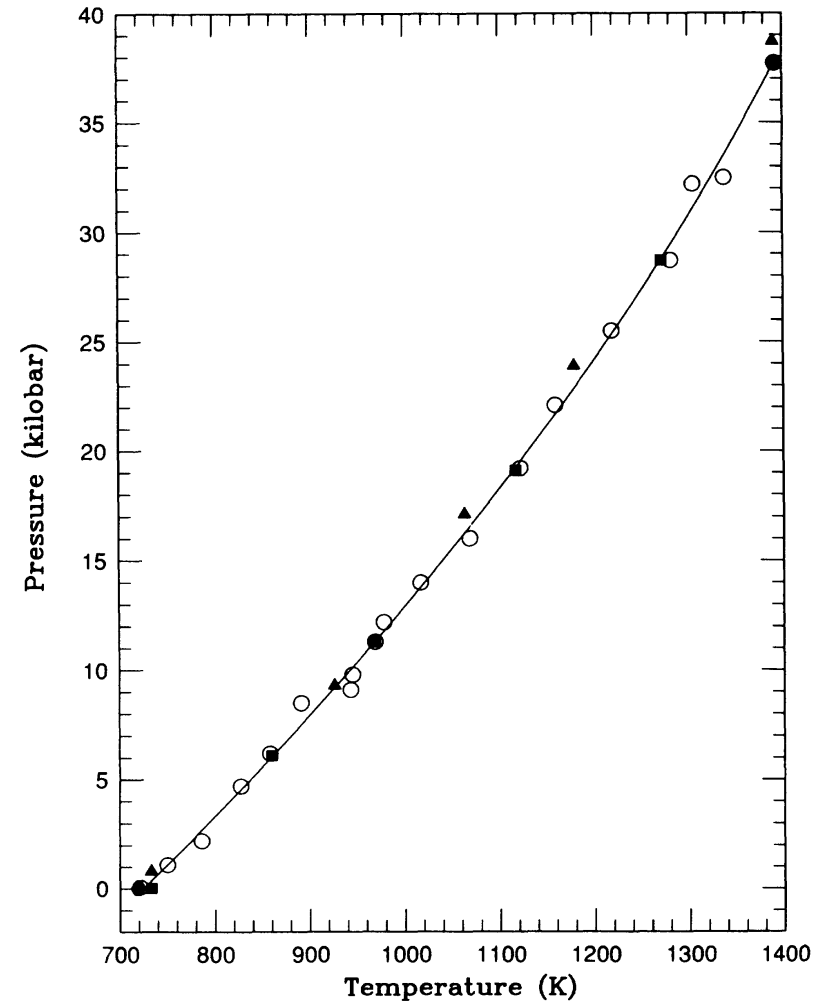

FIG. 4. $P-T$ phase diagram, as deduced from the simulations. Data points are from the simulations, listed in Tables I and II. The symbols indicate results for different system sizes: open circles, $N=1024$; solid squares, $N=4096$; solid triangles, $N=8192$; solid hexagon, $N=65536$. The solid line is a fit of the $N=1024$ results to the form $T=T_{0}+\alpha P+\beta P^{2}$; the $P=0$ melting point is found to be $T_{0}=724 \mathrm{~K}$. ing temperature is a common shortcoming of the EAM models. ${ }^{11}$

The dynamics of the solid-liquid interface was also examined in the paper by Mei and Davenport; it was found that at $T=700 \mathrm{~K}$ (below their calculated transition temperature of $800 \mathrm{~K}$ ) the crystal phase tended to grow, while at $T=900 \mathrm{~K}$ (above their transition temperature) the crystal began to melt. This approach is similar to ours, in that they examine a coexisting system in order to check their calculated transition temperature. Again, the argument is that the system will tend to solidify if the temperature is too low, and will liquify if the temperature is too high. However, instead of allowing the system to equilibrate, they simply observe the initial movement of the interface. Their results on the dynamics of the interface are consistent with our melting temperature of $724 \mathrm{~K}$, as well as the higher melting temperature they obtained. However, we consider this approach somewhat unreliable, as we commonly saw systems in which the crystal at one interface melted, while the liquid at the other interface crystallized. When this occurs, there is no way to state whether the system is too cool or too warm. This suggests that fluctuations in the boundary position make predictions based upon the boundary motion difficult. The possibility of melting at one end and solidification at the other is evident in Fig. 1(b), where some of the system that began in the crystal phase (open circles) is now melted, and some that began in the liquid phase (solid circles) is now solid. Such results are not surprising, as the fluctuations in the potential energy are largest near a phase transition. Also, the dynamics are also determined by local pressure equilibration; both the temperature and pressure must equilibrate. These processes are coupled, making the interpretation of the 
TABLE II. Same as Table I, but for simulations involving 4096, 8192, and 65536 particles.

\begin{tabular}{|c|c|c|c|}
\hline $\begin{array}{l}\text { Pressure } \\
\text { (kbar) }\end{array}$ & $\begin{array}{c}\text { Melting temperature } \\
(\mathrm{K})\end{array}$ & $\begin{array}{c}\text { Total energy/atom } \\
(\mathrm{eV})\end{array}$ & $\begin{array}{c}\text { Box size } \\
\left(\AA^{3}\right)\end{array}$ \\
\hline \multicolumn{4}{|c|}{4096 particles } \\
\hline 0.0 & 733 & -3.125 & $68.30 \times 33.60 \times 33.60$ \\
\hline 6.1 & 860 & -3.099 & $67.60 \times 33.35 \times 33.35$ \\
\hline 11.3 & 969 & -3.090 & $67.00 \times 33.00 \times 33.00$ \\
\hline 19.1 & 1117 & -3.055 & $66.55 \times 32.60 \times 32.60$ \\
\hline 28.7 & 1271 & -3.025 & $66.15 \times 32.00 \times 32.00$ \\
\hline 37.7 & 1392 & -2.980 & $65.80 \times 31.60 \times 31.60$ \\
\hline \multicolumn{4}{|c|}{8192 particles } \\
\hline 0.8 & 733 & -3.130 & $135.5 \times 33.6 \times 33.6$ \\
\hline 9.3 & 926 & -3.095 & $133.8 \times 33.2 \times 33.2$ \\
\hline 17.1 & 1063 & -3.070 & $132.2 \times 32.8 \times 32.8$ \\
\hline 23.9 & 1179 & -3.060 & $130.6 \times 32.4 \times 32.4$ \\
\hline 38.7 & 1390 & -3.000 & $128.2 \times 31.8 \times 31.8$ \\
\hline \multicolumn{4}{|c|}{65536 particles } \\
\hline 0.0 & 718 & -3.130 & $272.0 \times 67.2 \times 67.2$ \\
\hline
\end{tabular}

interface dynamics unclear. Therefore, we do not believe that monitoring the initial movement of a phase boundary is necessarily a good technique for determining the transition temperature.

The fit of the data shown in Fig. 4 indicates that the slope at $P=0$ is $d P / d T=43 \mathrm{bar} / \mathrm{K}$. The ClausiusClapeyron equation relates the slope to the latent heat $L$ and the difference between the specific volumes of the solid and the liquid phases:

$$
\frac{d P}{d T}=\frac{L}{T\left(v_{l}-v_{s}\right)} .
$$

Using this along with the density of the different phases, we could estimate the latent heat of the transition at zero pressure. Alternatively, noting that $v_{l}-v_{s}$ is very weakly dependent upon temperature and pressure, we may calculate the change of entropy $\Delta S \equiv L / T$ that occurs at the transition. This will be nearly constant throughout the linear regime (i.e., over the temperature range where $d P / d T$ is nearly constant). We found that at $P \approx 0$, the specific volumes in the liquid and solid phases were approximately $v_{l}=20.5 \AA^{3}$ and $v_{s}=16.6 \AA^{3}$, resulting in a value of $\Delta S=1.2 k_{B}$. This is identical to the value of $\Delta S=1.2 k_{B}$ calculated using the solid- and liquid-phase energies given by Mei and Davenport at their calculated melting temperature $T_{m}=800$, and close to the experimental value of $\Delta S=1.4 k_{B}$.

\section{DISCUSSION}

In this paper, we have calculated a portion of the $(P, T)$ phase diagram of an EAM model of aluminum. This work demonstrates that the melting line of a model system may be accurately and systematically determined by using constant energy, constant volume molecular dynamics to simulate coexisting liquid and solid phases, and allow- ing the system to equilibrate. The melting point for this model was previously determined ${ }^{8}$ to be near $T_{m}=800$ $\mathrm{K}$ at $P=0$; we find a somewhat lower transition temperature of $T_{m}=724 \pm 10 \mathrm{~K}$. We calculated the change in entropy at the transition to be $\Delta S=1.2 k_{B}$, using the Clausius-Clapeyron equation, and found the same value using the results of Mei and Davenport. ${ }^{8}$ This value is in reasonable comparison with the experimental value of $\Delta S=1.4 k_{B}$.

This technique has several advantages over other methods. The most important advantage is that the simulated system does not have to undergo the nucleation process in order to transform; normally, the slow nucleation time is the source of the hysteresis in "computer experiments" in which a crystal is heated until it transforms into a liquid. Secondly, unlike techniques based upon Eq. (1) which use a series of simulations to determine the free-energy difference between the system and a solvable system, the coexistence technique can, in principle, reveal a melting point in a single simulation. In fact, once several points on the phase diagram were known, we found it easy to predict the simulation conditions that would reveal a new point on the phase diagram. Thus, the total number of simulations performed was not much greater than those listed in Tables I and II. In contrast to this, Mei and Davenport ${ }^{8}$ required 10 simulations simply to determine the free energy of the liquid phase of aluminum at a single temperature and pressure.

While we believe that this approach is very useful, it is important to be aware of possible difficulties that can arise. First, as this is based upon a finite-size system, there is no true transition point. In principle, because of this, there will be a small range of temperatures over which coexistence may occur. In practice, for the number of particles used in our simulations, the actual behavior will be very similar to that of a true transition, to the point of being indistinguishable in the simulations.

More importantly, changing the size of the system 
can have the effect of shifting the apparent transition temperature, ${ }^{17}$ and thus the phase diagram may have a systematic error due to these finite-size effects. However, these effects are usually quite small for first-order transitions, due to the finite correlation length as the transition is approached; the effects vanish rapidly with increasing system size. In our case these effects are certainly smaller than the scatter in the results shown in Fig. 4: increasing the size of the system from 1024 particles to 4096 reduced the scatter, but did not noticeably change the results. This is demonstrated by the fact that the data from the $N=4096$ simulations agreed with the fit to the $N=1024$ data. Increasing the size further, by doubling the dimension of the $N=4096$ system perpendicular to the interface, appears to slightly decrease the observed transition temperatures, as can be seen in Fig. 4. However, at high pressures (where this shift is largest), this change is no more than $1 \%$. This is less than our estimated error, and (given the accuracy of the model) is insignificant. A further doubling of all dimensions did not produce a significant change in the $P=0$ results, as indicated in Table II for the $N=65536$ system. Thus, for all sizes, we find that the $P=0$ results are within the range $T=724 \pm 10 \mathrm{~K}$.

Thus, although changes in the geometry and in the number of particles could, in principle, affect the observed transition temperature, our results show no such finite-size effect. We expect that the shift in the transition temperature due to the system size will be small in most solids near their melting temperature, and that it is not necessary to go to extremely large numbers of particles to extract accurate information. (Note that this will not be true for two-dimensional melting, where fluctuations over long distances can occur. This can cause important finite-size effects, making the bulk limit difficult to extract. ${ }^{18,19}$ )

The most important size effect is that the system must be large enough to allow for equilibration, which may involve a significant fraction of the system transforming. Clearly, the system should not entirely transform - this would indicate that the system is not at an equilibrium transition point. To check the possibility that this could affect our results, we performed a series of simulations with a total of 512 particles, half as many as used to obtain the results for the 1024-particle system. The geometry in the $y$ and $z$ directions were the same, but the length along the $x$ direction was halved. In that case, the system tended to transform completely; further, over a range of energies, the system would either become completely liquid or completely solid in an unpredictable fashion. Thus it is very important that this technique be used with a sufficient system size and simulation time to ensure that the system is in a true equilibrium coexistence regime.

The requirement of such a large system may currently make it difficult to apply this technique to $a b$ initio and tight-binding techniques; however, increasingly powerful computers and recent developments in calculational techniques ${ }^{4,5,20}$ may soon make this a practical technique even for these more realistic techniques. Our work clearly demonstrates the advantages of this approach: its simplicity, speed, and immediate applicability make this technique a powerful tool for the theoretical exploration of phase diagrams.

\section{ACKNOWLEDGMENTS}

J.R.M. would like to thank Dr. David Turner for his help and patience with implementing this work on the nCUBE and Paragon computers. Part of this work was made possible by the Scalable Computing Laboratory, which is funded by Iowa State University and Ames Laboratory. Ames Laboratory is operated for the U.S. Department of Energy by Iowa State University under Contract No. W-7405-Eng-82. This work was supported by the Director for Energy Research, Office of Basic Energy Sciences, and the High Performance Computing and Communications initiative. Some of this work was performed on the nCUBE 2 at Sandia National Laboratories.
${ }^{1}$ M.S. Daw and M.I. Baskes, Phys. Rev. Lett. 50, 1285 (1983); Phys. Rev. B 29, 6443 (1984).

${ }^{2}$ M.W. Finnis and J.E. Sinclair, Philos. Mag. A 50, 45 (1984).

${ }^{3}$ C.Z. Wang, K.M. Ho, and C.T. Chan, Comput. Mater. Sci. (to be published).

${ }^{4}$ X.-P. Li, R.W. Nunes, and David Vanderbilt, Phys. Rev. B 47, 10891 (1993).

${ }^{5}$ F.Mauri, G. Galli, and R. Car, Phys. Rev. B 47, 9973 (1993).

${ }^{6}$ M.C. Payne, M.P. Teter, D.C. Allan, T.A. Arias, and J.D. Joannopoulos, Rev. Mod. Phys. 64, 1045 (1992).

${ }^{7}$ D. Frenkel and A.J.C. Ladd, J. Chem. Phys. 81, 3188 (1984).

${ }^{8}$ J. Mei and J.W. Davenport, Phys. Rev. B 46, 21 (1992).

${ }^{9}$ J.Q. Broughton and X.P. Li, Phys. Rev. B 35, 9120 (1987).

${ }^{10}$ B. Widom, J. Chem. Phys. 39, 2808 (1963).

${ }^{11}$ S.M. Foiles and J.B. Adams, Phys. Rev. B 40, 5909 (1989).

${ }^{12}$ P. Stoltze, J.K. Nørskov, and U. Landman, Phys. Rev. Lett.
61, 440 (1988).

${ }^{13}$ F. Ercolessi, O. Tomagnini, S. Iarlori, and E. Tosatti, in Nanosources and Manipulation of Atoms under High Fields and Temperatures: Applications, edited by Vu Thien Binh, N. Garcia, and K. Dransfeld, NATO ASI Series E, Vol. 235 (Kluwer, Dordrecht, 1993), p. 185.

${ }^{14}$ G. Bilalbegovič, F. Ercolessi, and E. Tosatti, Surf. Sci. Lett. 258, L676 (1991).

${ }^{15}$ G. Galli, R.M. Martin, R. Car, and M. Parinello, Nature (London) 250, 1547 (1990).

16 J.R. Morris (unpublished).

${ }^{17}$ J.R. Morris and R.J. Gooding, Phys. Rev. B 43, 9733 (1992).

18 J.A. Zollweg and G.V. Chester, Phys. Rev. B 46, 11187 (1992).

19 J. Lee and K.J. Strandburg, Phys. Rev. B 46, 11191 (1992).

${ }^{20}$ S.-Y. Qiu, C.Z. Wang, K.M. Ho, and C.T. Chan (unpublished). 